\title{
Germanica
}

\section{L'autobiographie comme « exécution de soi- même » : Franz Fühmann: Zweiundzwanzig Tage oder die Hälfte des Lebens}

Die Autobiographie als «Exekution seiner selbst»: F. Fühmanns, Zweiundzwanzig Tage oder die Hälfte des Lebens

\section{Anne Lagny}

\section{OpenEdition Journals}

Édition électronique

URL : http://journals.openedition.org/germanica/1924

DOI : 10.4000/germanica.1924

ISSN : 2107-0784

\section{Éditeur}

Université de Lille

\section{Édition imprimée}

Date de publication : 30 juin 1997

Pagination : 95-109

ISBN : 9782098426320

ISSN : 0984-2632

\section{Référence électronique}

Anne Lagny, «L'autobiographie comme « exécution de soi-même »: Franz Fühmann: Zweiundzwanzig Tage oder die Hälfte des Lebens », Germanica [En ligne], 20 | 1997, mis en ligne le 24 juin 2013, consulté le 06 octobre 2020. URL : http://journals.openedition.org/germanica/1924 ; DOI : https://doi.org/ 10.4000/germanica.1924

Ce document a été généré automatiquement le 6 octobre 2020.

(c) Tous droits réservés 


\section{L'autobiographie comme « exécution de soi-même »: Franz Fühmann: Zweiundzwanzig Tage oder die Hälfte des Lebens}

Die Autobiographie als «Exekution seiner selbst»: F. Fühmanns, Zweiundzwanzig Tage oder die Hälfte des Lebens

\section{Anne Lagny}

1 Je dédie ce texte à la mémoire de Florence Khodoss

2 En 1972, Fühmann effectue un séjour à Budapest à l'invitation du Pen Club hongrois et en rapporte un journal qu'il publie sous le titre de Zweiundzwanzig Tage oder die Hälfte des Lebens. On s'arrêtera à ce titre, qui articule dans un rapport d'équivalence deux dimensions temporelles, deux projets d'écriture dont la compatibilité est pour le moins problématique : les "vingt-deux jours $»^{1}$ nous orientent vers le journal, en mettant l'accent sur la séquence chronologique caractérisée par la succession des moments et la durée brève ; la « moitié de la vie » renvoie à la longue durée de l'existence individuelle, saisie en un point privilégié où viennent se nouer les trois dimensions du passé, du présent et dix futur².

3 L'entreprise autobiographique postulée dans le titre se constitue ici non par la rétrospection, mais par la médiation d'une autre temporalité, celle de la quotidienneté du journal. Fühmann n'a pas voulu réduire la disjonction entre ces deux perspectives : le voyage à Budapest n'est pas le retour d'un pèlerin sur les lieux de son passé, théâtre «naturel » d'une remémoration activée par le décalage temporel, mais un départ dans l'inconnu, puis l'installation laborieuse et précaire dans un ailleurs. La «moitié de la vie » ne se donne pas d'emblée comme un bilan lisible, mais se découvre au terme d'un trajet où doivent se rejoindre, sinon coïncider, les deux strates temporelles, la moitié de la vie devenant ainsi l'horizon et le sens de ces vingt-deux jours ${ }^{3}$. 
4 La question de savoir comment le journal rejoint l'autobiographie est plus complexe qu'il n'y parait tout d'abord. La lecture du texte se présente à bien des égards comme une épreuve où l'auteur s'applique à décevoir les attentes communes. Le projet d'un modeste carnet de voyage, qui ne se distingue pas par nature du journal qu'il tient quotidiennement, est censé se réaliser sans le concours actif de l'auteur ou sa volonté de mettre en forme, et qui plus est, il relativise encore la fragile unité thématique qui pourrait se constituer autour de ce voyage à Budapest ${ }^{4}$. Dans vingt-deux sections d'inégale longueur, les instantanés sur la ville étrangère se mêlent aux réflexions personnelles impromptues : notations fugitives, anecdotes, citations, extraits du guide touristique, listes entières (celle d'une carte des desserts, celle des étalages du marché), souvenirs de lectures, journal de l'écrivain réfléchissant sur les mots, du traducteur aux prises avec une langue étrangère qu'il renonce à maitriser parfaitement... L'ensemble frappe par son caractère allusif, décousu, fragmentaire : c'est un montage de matériaux disparates, à divers degrés d'élaboration, un collage qui juxtapose différentes strates de perception, de réflexion, et affirme son caractère ouvert comme condition de son écriture. Comment se situer vis-à-vis de cette œuvre qui semble oublier son lecteur, tout en maintenant, paradoxalement, à la frontière de la lisibilité, la possibilité d'une véritable relation de lecture $?^{5} \mathrm{~L}$ 'immersion dans l'atelier de l'écrivain est une étape dans le parcours qui constitue le sens de cette œuvre caractérisée par la difficulté même de se constituer en œuvre, et dont les enjeux n'apparaissent pas tout d'abord.

5 La première séquence, qui fait passer sans transition perceptible de la situation présente - la gare, quelques instants avant le départ du train -, à la vision d'un paradis nocturne - tous les animaux de la création endormis dans la paix-, crée une impression de perméabilité entre extérieur et intérieur et suggère les multiples dimensions de l'expérience mentale, qui va et vient entre les instantanés de la perception présente et les échos renvoyés par la conscience, l'esprit au travail ou la mémoire. La tentative de fixer les coordonnées d'un espace-temps personnel dans le moment du départ aboutit au flottement de tous les repères: l'espace est un entre deux, le temps vécu cesse de coïncider avec la mesure de l'horloge. Dans le suspens de l'heure zéro, l'illusion berceuse d'un monde endormi dans une tranquillité paradisiaque s'efface d'un coup devant le surgissement d'un univers de hantises ${ }^{6}$. Le journal se constitue entre les deux versants nocturne et diurne de l'expérience humaine; entre l'angoisse informe et la volonté de figuration. C'est ainsi qu'à la vision fugitive du monde d'en bas, comme instant soustrait à la durée, succède la représentation géométrique de l'instant comme point d'intersection: la projection dans l'espace des lignes et des plans détourne le regard de la confrontation avec l'informe ${ }^{7}$.

6 Le « je » qui fait ici une entrée discrète n'a pas la consistance du sujet qui est le noyau organique de l'autobiographie. Il se définit comme un foyer de perception et de réflexion, dans un rapport d'évidence et d'opacité à lui-même : évidence de l'activité mentale, opacité de son identité. La première séquence du journal n'installe pas le «je » au centre de la page, elle commence par fixer l'impression en image, le «je» n'intervenant qu'après-coup, pour y ajouter l'écho de l'intellect ou de la mémoire. La situation de ce « je », qui s'affirme progressivement comme l'enjeu essentiel du journal, commence ici à s'esquisser dans la dialectique entre la surface et la profondeur. Où se trouve-t-il entre surface et profondeur? de ce point de vue, que peut-il réfracter du monde qui l'entoure, saisir ou projeter de lui-même? Ces questions fondamentales de l'écriture autobiographique, Fühmann n'y apporte pas de réponse générale. Il les rabat 
sur sa situation présente d'étranger dont il tire parti pour définir un mode d'approche particulier, en s'imposant de ne pas réduire ses difficultés d'accès au continent largement inconnu de la civilisation hongroise et de marquer la distance qui le sépare à jamais d'un monde attirant, mais irrémédiablement autre. C'est donc dans la différence avec sa culture d'origine que l'étranger, le demi-touriste, va trouver ses premières prises : le goût d'une culture de la vie et de la couleur, contre l'austérité germanique.

7 Cette appréhension de la réalité par l'extérieur et la surface est l'inverse de la démarche suivie dans Das Judenauto. Ce recueil autobiographique, antérieur d'une dizaine d'années au journal de Budapest, s'ouvre sur la question «Wie tief hinab reicht das Erinnern?», qui oriente vers l'exploration de la mémoire. Or, ce parcours a échoué, de fait, à exorciser définitivement les souvenirs du passé, comme en témoigne la vision fugitive du minuit, au début du journal. Le pari sur la surface, sur lequel se construit le journal de Budapest, apparaît ainsi comme un relais nécessaire permettant de dépasser les limites du premier texte autobiographique, en dégageant les leçons de cette forme d'échec.

8 L'auteur prend le parti de se concentrer sur sa situation présente d'étranger, condamné à rester à la surface des choses : c'est la seule réalité qui lui soit accessible, et son premier terrain d'investigation. Ce pari, malgré une limitation qui lui est inhérente, n'est pas une démission: il implique la mobilisation de l'esprit et de ses ressources d'analyse, à partir du postulat que l'étude et l'épreuve de la surface orientent vers la profondeur et sont une voie d'accès à l'être ${ }^{8}$. Un premier exemple nous en est donné, quelques paragraphes plus loin, avec l'abandon aux impressions sensibles, visuelles, auditives, gustatives : la description appuyée du goût de la soupe de poisson, prélude à la célébration de la cuisine hongroise, fixe l'instant de la jouissance comme repère d'une dimension où le sujet se retrouve, naturellement, à l'unisson du monde extérieur.

9 Le journal suit la ligne discontinue des impressions, qui lancent de frêles passerelles entre la réalité extérieure colorée et une réalité intérieure opaque et inaccessible. Le sujet, disponible à ce qui l'affecte, s'abandonne aux hasards du parcours et se laisse distraire par ce qui se présente dans l'instant. L'esprit en fait l'occasion de sa réflexion et commence à élaborer son matériau, qu'il abandonne souvent provisoirement à son inachèvement, faute d'un outillage adéquat. L'épreuve de l'écho de la mémoire, qui trace la ligne de partage entre l'élément propre et l'extériorité, décisive au début de Das Judenauto, trouve son équivalent ici dans l'épreuve de la résonance. L'enjeu en est la découverte du point qui marque l'origine personnelle de l'écriture propre, celle qui ne se confond avec aucune autre et justifie la conscience de l'auteur de remplir sa "fonction partielle ", écrire ce que nul autre ne peut écrire. La conquête est ici plus malaisée, car la présence du sujet à lui-même n'est donnée que par le biais des impressions actuelles qui décentrent l'écriture et la détournent temporairement du sujet et de son histoire. Un épisode du début du journal marque nettement cette réserve. La découverte du panorama de Budapest fait surgir le souvenir de sa captivité dans le Caucase et l'image du Hongrois qui lui reprochait la destruction du Pont Elisabeth, l'un des plus beaux. Ce rappel épisodique n'est pourtant pas l'occasion d'amorcer le récit autobiographique, mais bien de préciser son sentiment actuel - c'est la première fois qu'il évoque ce souvenir sans malaise - et son incompréhension d'étranger devant l'attachement sentimental des Hongrois aux ponts de Budapest ${ }^{9}$. Un de ses amis, devant qui il mentionne l'anecdote, le presse de transmettre ce témoignage aux générations futures, ce que l'écrivain accueille avec détachement ${ }^{10}$. Si Das Judenauto 
est inspiré par le devoir de témoigner, l'auteur du journal, lui, s'est délié de l'obligation crispée de rendre des comptes de son passé. Le séjour à Budapest marque un temps de relâche, et l'occasion de retrouver la libre disposition de soi, en même temps que de nouvelles ressources de figuration, nécessaires à la poursuite de sa création. Il s'agit en premier lieu de réintroduire l'espace de la vie, non de la thèse, dans la littérature ${ }^{11}$.

Dans cette perspective, on comprend que les impressions ne se résolvent pas immédiatement en signifiants de la réalité intérieure personnelle. Le rapport entre les choses de la surface et l'être intérieur est un rapport d'indication (hinweisen), non d'équivalence. Le lecteur est contraint de suivre la ligne discontinue du journal et ne s'oriente, à la longue, qu'en discernant dans ce flux (différent du flux de conscience, par l'acte réflexif d'écrire) des noyaux thématiques, des écheveaux de réflexion dont les fils ne sont pas encore démêlés et dévidés. Comme celui qui écrit, il doit attendre que l'esprit se fraie obscurément un chemin, retrouve des pistes dont la trace est profondément enfouie ou affleure à peine à la surface des choses, à partir de ce que lui donne le hasard. Un hasard qu'il organise en parcours à partir de ses propres possibilités, mais aussi de ses hésitations et de ses impuissances. La résonance, l'écho de la conscience sera ici le signe de l'importance de l'objet saisi, sans garantir, d'ailleurs, l'accès immédiat à la profondeur. Les hasards peuvent (et cela non nécessairement) fonctionner comme jalons de pistes possibles, dont le parcours éprouvera la viabilité ou l'impraticabilité.

11 Le journal traite la surface et traite en surface, ce qui peut s'entendre et se réaliser de différentes manières, qui constituent autant de ressources nouvelles à expérimenter. Tout d'abord, l'écrivain fournit un équivalent visuel de l'appréhension de la réalité par la surface en matérialisant les feuillets qu'il écrit. Le livre a conservé deux typographies différentes, les caractères imprimés et ceux de la machine à écrire, ce qui introduit dans le texte définitif l'étape du manuscrit. La discontinuité des impressions de surface trouve son équivalent dans les blancs du journal et l'alternance des typographies, ainsi que dans les passages abrupts d'une forme à une autre. On citera, comme particulièrement significatifs, les exercices de poésie concrète et les récits de rêves, tous deux transcrits en caractères dactylographiés. Les premiers, qui correspondent à un moment d'oisiveté, soulignent, dans l'instant où ils le franchissent sans le combler, le temps et l'espace d'un vide dont ils détournent l'esprit : le calligramme convertit la feuille en espace pictural, la rendant ainsi à sa nature de surface. Les rêves, qui s'inscrivent dans le corps du texte comme énigmes à résoudre, figurent la surface de la profondeur. Le journal s'élabore comme montage de ces différents reliefs.

On remarquera la propension de l'auteur à recourir à l'abstraction pour formaliser un problème, en construire la figure géométrique ou l'équation à partir de données mathématiques ou logiques. La formulation d'hypothèses ou de raisonnements par analogie permet d'approcher une réalité intérieure qui se dérobe. Dans le même ordre d'idées, on remarquera l'attention soutenue aux phénomènes de langue, particulièrement aux caprices de l'usage ${ }^{12}$. Ces exercices épisodiques ont pour fonction de débusquer l'arbitraire qui occulte la signification profonde des mots, de rendre ceux-ci à leur résonance première. Ils contribuent ainsi à une régénération de la langue et à une mobilisation nouvelle de ses ressources.

Pas plus que le sujet, les ressources de la figuration traditionnelle ne sont immédiatement disponibles. Dans l'approche du pays étranger, l'énumération se substitue fréquemment à la description. Lorsqu'il y a description, celle-ci ne se 
développe jamais dans le sens du panorama, elle reste au niveau de la notation, à la recherche d'images qui fixent et transposent une impression visuelle ou sonore. Ce refus des conventions ordinaires de l'écriture « littéraire » correspond à la délimitation stricte d'un champ de perception, qui apparaît progressivement comme déterminé par une expérience et une histoire singulières. L'écriture de la surface renvoie donc, en creux, à celui qui écrit, en traçant au plus juste le périmètre qui circonscrit sa tâche d'écrivain. Chaque séquence est pour l'auteur l'occasion de réfléchir sur ses ressources propres, en assumant les limites ou échecs de la figuration en tant que tels. C'est ainsi par exemple que l'auteur, se promenant dans le quartier populaire du $8^{\mathrm{e}}$ arrondissement de Budapest, est témoin d'une scène de rue dont il enregistre les détails sans pouvoir l'interpréter (pour lui comme pour le lecteur), du fait de sa maitrise imparfaite de la langue. Réfléchissant sur cette limite de son pouvoir d'écrivain, il la met en relation avec sa situation d'étranger dans la ville de Budapest, mais aussi avec ses propres origines, bourgeoises, qui l'éloignent de la veine populaire d'un Mándy, auteur familier de ce quartier, capable d'utiliser ce motif ${ }^{13}$.

Cette écriture, apparemment capricieuse et libre, est donc entravée par des obstacles qui ne sont pas toujours discernables à première vue, et qui tiennent à l'intime solidarité entre l'écriture et l'état de la conscience. La réticence à utiliser les ressources de la figuration littéraire traditionnelle s'explique par la méfiance vis-à-vis de celles-ci, sources possibles de déviation ou d'imposture. Seul le détour permet de respecter l'impulsion authentique de cette écriture, sans en épuiser précocement l'énergie et sans la dévier. La surface, qui dans un premier temps semble annuler la profondeur, préserve au contraire ses chances de se manifester, elle en réserve l'espace. Dans un entretien, Fühmann compare l'écriture de Zweiundzwanzig Tage... au moment où se débouche une canalisation gelée : écoulement difficile, puis jaillissement torrentiel, enfin régulation du flux ${ }^{14}$. Cette image suggère de chercher le sens du journal dans la dynamique du courant qui se libère, sans s'arrêter aux impuretés charriées par le torrent dans un premier temps. L'état transitoire d'une pensée prise dans le gel a été néanmoins conservé, sans doute parce qu'il ne peut être dépassé que dans ce processus d'expulsion. Il ne convient donc pas d'assigner un sens précis et immuable à des formulations approximatives, qui ne sont pas retouchées. L'accent est mis ici non sur la formule figée, mais sur la remise en mouvement du processus de l'écriture.

Le journal, sous cette forme lâche, qui affranchit le narrateur de contraintes extérieures, préserve la proximité du sujet à lui-même. Cet enjeu majeur demeure latent, avant d'être brutalement révélé par l'irruption de la maladie, qui marque le moment de la crise du journal, la menace de l'éclatement ou de la dispersion du sujet. Lagrippe qui terrasse le narrateur quelques jours après son arrivée a pour effet de le recentrer sur lui, en ménageant l'accès à la profondeur par l'expérience de sensations nouvelles. La crise se résoud par un moment de désorientation qui fait écho à celui du départ et l'amplifie. Les heures passées dans le Lukas-Bad, qu'on lui a conseillé comme une thérapie efficace, prennent dans ce contexte la valeur d'un périple initiatique, même si l'épisode reste ancré dans la situation concrète du touriste égaré, spectateur distancié, voire amusé, de ses errances labyrinthiques à la recherche de l'entrée, de la bonne salle, de sa cabine, de ses affaires, de la sortie. Le lecteur attentif sera sensible à la dimension symbolique qui s'esquisse ici par simples touches, suffisantes pourtant pour suggérer l'horizon mythique de cette scène réaliste à la frange du fantastique : le dépouillement des vêtements qui renvoie l'homme à sa nudité originelle, celle de la naissance et de la mort ; la vision des clients en aréopage de tous les héros des légendes 
hongroises à l'instant d'un jugement dernier ${ }^{15}$. Ce bain marque l'instant d'une régénération symbolique qui le rend à lui-même, c'est-à-dire favorise l'émergence des interrogations existentielles occultées ou éludées jusqu'alors. Deux jours plus tard, défiant toutes règles de prudence, l'auteur sort pour précipiter son rétablissement (ou une rechute), et c'est à cet instant, à la frontière entre la maladie et la santé, au bord du fleuve, devant le panorama de Budapest, qu'il tente d'embrasser ce que fut la moitié de sa vie. Ce geste, qui n'est pas sans rappeler le début de l'autobiographie de Stendhal, Vie de Henry Brulard, est à peine ébauché - l'auteur est distrait, une fois de plus, par le spectacle de la rue -, mais la dimension propre de l'autobiographie, à défaut de sa réalisation, vient s'inscrire dans l'œuvre. La question principale n'est pas ici : «Qui suis-je ? », mais « qu'ai-je produit? »16

16 Le retour à la santé fournit, en tant qu'expérience immédiate, la matrice d'une réélaboration du matériau personnel, l'approfondissement de la réflexion abstraite sur le même et l'autre, amorcée par la remarque sur l'absence de majuscule à «ander», qui débouche sur le complexe de la métamorphose. La réflexion sur la notion de Wandlung, centrale dans son itinéraire personnel, se substitue à la remémoration ${ }^{17}$. Le journal de Budapest, en effet, ne parvient pas à donner une consistance véritable aux rares souvenirs qu'il mentionne. Cette réticence est l'indice d'un problème qui apparaît avec la progression de la réflexion sur l'existence humaine. Les souvenirs en effet ne sont pas inertes, certains reviennent au jour et viennent se fondre dans l'expérience, mais d'autres, impossibles à rappeler, demeurent terra incognita, entrevue dans le rêve, mais pratiquement inaccessible, sinon au prix d'une odyssée peut-être fatale à qui l'entreprend et peut succomber sous la poussée de cette réalité refoulée. Le journal hésite visiblement ${ }^{18}$, puis renonce définitivement à l'exploration des profondeurs ultimes.

La réflexion sur la notion de Wandlung ${ }^{19}$ n'en permet pas moins le retour sur l'existence personnelle, tandis que le problème de sa figuration pose la question du projet littéraire et de l'œuvre réalisée. C'est ainsi qu'apparaissent les insuffisances de Das Judenauto ${ }^{20}$. Pour l'homme, pour l'intellectuel, il s'agit au fond de penser non la disjonction entre l'état antérieur et l'état ultérieur, mais l'articulation de l'identité et de la transformation; pour l'écrivain, il s'agit de transcrire la perception et la compréhension de ce changement. Sur cette voie, Fühmann se heurte à la contradiction entre son apprentissage socialiste, qui lui a fait endosser les vêtements de l'esthétique réaliste, et la consistance de sa problématique intérieure, celle de l'ancien nazi doutant d'être profondément transformée ${ }^{21}$. Or, le modèle du Bildungsroman du réalisme socialiste, qui privilégie les résultats positifs d'une transformation par étapes codifiées, occulte totalement la question même de cette transformation et de l'individu dans lequel elle s'accomplit. Le modèle de la conversion, celle d'Augustin ou de Saul de Tarse, qui pourrait fournir un schéma de compréhension et de figuration de la genèse du mal, est également rejeté par l'auteur. S'il donne un équivalent sensible de certaines expériences fondatrices, il n'en élucide pas la nature. Dans le modèle religieux, c'est un autre qui assume le passage de l'homme ancien à l'homme nouveau. L'homme régénéré par Dieu ne conserve plus en lui la trace de l'homme qu'il a été et qu'il ne comprend plus, même s'il se souvient.

18 La tâche de comprendre cette transformation, et surtout de la figurer, ne peut donc se définir qu'en dehors de cadres - esthétique du réalisme socialiste, modèle de conversion - qui réduisent ou nient l'expérience singulière de la contradiction et de la 
fracture. Ce qui serait de moindre conséquence existentielle, sinon esthétique, dans le cas d'un parcours individuel banal, devient un enjeu vital pour un homme confronté à son passé nazi et à la faute collective inexpiable à laquelle il a part ${ }^{22}$. Cet horizon indépassable interdit l'élucidation totale de son passé et pose des obstacles presque insurmontables à sa représentation adéquate. Celle-ci supposerait en effet de prendre la pleine mesure de ce que Fühmann appelle l'Un et l'Autre dans la réalité humaine commune, la part de l'humanité et de l'inhumanité dont il s'agirait de délimiter la frontière, qui passe à l'intérieur d'un même moi. Comment comprendre l'Un et l'Autre, celui qu'il a été, celui qu'il est devenu, dans l'unité de la vie d'un seul et même (et autre ?) homme? L'un et l'autre sont si inextricablement liés que la tentative de les dissocier est vouée à l'échec. L'auteur ne pourrait sortir de cette impasse que par le suicide, qui reviendrait à tuer en lui l'un et l'autre.

C'est à ce point que l'auteur se heurte à l'illisibilité de son parcours et à l'impossibilité de poursuivre son œuvre dans la voie de l'autobiographie. Entre la figure extérieure de son parcours et la question de l'orientation de son existence, il y a disjonction absolue. Dans les faits, le jeune nazi, affecté par le jeu des circonstances au service des communications dans les troupes d'occupation en Grèce, était éloigné des théâtres d'opération où ont été commis les crimes contre l'humanité. Sa responsabilité effective serait donc dégagée. Mais le hasard objectif laisse subsister l'interrogation torturante sur la possibilité de commettre de tels crimes ${ }^{23}$. La chronologie de la vie d'un homme ne fournit pas de structure signifiante à hauteur des enjeux de cette tentative d'autobiographie, car elle est affectée du signe du hasard, et ne permet donc pas d'élucider l'ensemble des déterminations profondes qui ont affecté l'existence individuelle.

20 Si l'auteur ne peut imposer la perspective de la rétrospection, la dimension du temps n'en demeure pas moins présente ici comme la conscience d'un écoulement irréversible, l'urgence du bilan, l'angoisse du temps compté. Le «milieu de la vie» déchire ce journal, en rappelant l'urgence de définir sa situation, de remplir sa fonction d'écrivain, du cœur même de l'angoisse existentielle. Il est symbolisé par l'ombre du diable, qui surgit à deux endroits stratégiques du livre : en prélude à l'ouverture de la dimension autobiographique, et à la fin du séjour à Budapest. Le voyage est bien près de s'achever sur une défaite de l'homme, de l'écrivain ${ }^{24}$. Il suffit pourtant d'une rencontre fortuite pour que l'apparition s'évanouisse. Nous retrouvons ici, avec les impressions de la dernière soirée, le dernier repas, les adieux, la dernière figure de la dialectique entre surface et profondeur qui structure le journal. La surface discontinue de la réalité extérieure, avec ses surprises, ses occasions, distrait de la confrontation avec la profondeur, elle permet au sujet de se reprendre, temporairement ${ }^{25}$.

21 L'écriture laisse irrésolue l'interrogation existentielle, mais fraie la voie d'une réalisation de soi par l'accomplissement d'une fonction partielle dans la littérature. Une fois posée et écartée la tentation du suicide et de la suppression de l'Un et de l'Autre - la part d'inhumanité et d'humanité -, il reste l'alternative de transposer l'exigence de s'exécuter soi-même ${ }^{26}$ dans la dimension d'un agir réflexif retourné sur le sujet et non sur un objet, le champ même de la littérature. Si l'homme ne peut pas devenir totalement un autre, il se régénère par son écriture, dans le moment où celle-ci semble se déstructurer. Fühmann, évoquant dans une lettre à un ami l'importance du journal de Budapest, affirmait que celui-ci lui avait ménagé l'accès à la littérature. La scène dans le Lukacsbad est la figure de cette régénération de la personne, comme de 
l'œuvre qui commence à quitter les chemins de la figuration traditionnelle ${ }^{27}$ pour s'ouvrir à la dimension du mythe ${ }^{28}$ Dans son exploration de ressources nouvelles, Fühmann rencontre les écrivains qui posent pour lui d'importants jalons de sa propre création : Goethe pour la réflexion sur le symbole et l'image, Jean-Paul pour le rêve, les mythes, Hoffmann et Gogol pour le fantastique, puis Rilke, Trakl, les écrivains refoulés par le socialisme, sans oublier le panthéon des écrivains hongrois qu'il traduit. A leur manière, tous figurent la situation de l'écrivain et la mise en forme d'une expérience humaine, contribuant ainsi à définir le lieu où s'ancre l'écriture et la forme de l'œuvre à venir. L'important, ici, est de trouver non une nouvelle forme de vie, comme dans Das Judenauto, mais une nouvelle forme de littérature, liée à une expérience singulière. L'écriture, pour se comprendre, fait appel à une dimension qui lui est extérieure, celle de l'expérience humaine, et c'est cette dimension de l'humanité commune qui est le sceau de l'authenticité de cette œuvre et de sa transmissibilité.

\section{NOTES}

1. - Chiffre que Hans Richter, dans son livre sur Franz Fühmann, suggère d'interpréter comme allusion cryptée à la date de naissance de l'auteur, cf. Franz Fühmann, ein deutsches Dichterleben, Berlin ; Weimar : Aufbau-Verlag, 1992.

2. - Ce pourrait être la perspective de l'autobiographie classique, cf. infra, note 16.

3. - «Die Hälfte des Lebens» évoque le poème de Hölderlin qui porte ce titre, dont Fühmann inscrit la référence, comme bien d'autres, en filigrane de son texte.

4. - «Ein Büchlein Reisenotizen soll werden, irgendetwas Loses, Buntes, nicht einmal auf Ungarn beschränkt, ein bißchen erweitertes Tagebuch, und das führe ich ohnehin jeden Abend» p. 15 (c'est moi qui souligne). L'emploi du neutre et de l'indéfini est ici fort expressif.

5. - «Könnte man eine Straße, ein Viertel, eine Stadt, ein Land durch die Gedanken, Träume, Erinnerungen, Gefühle beschreiben, die einem in ihrem Bannkreis kommen? Sähe ein Zweiter aus solchen Aufzeichnungen jene Stätten, erkennte er sie wieder, oder könnte er sich ein Bild von ihnen machen? Ein topographisches Abbild gewiß nicht, aber vom Geist des Ortes sagte solch ein Ideennotizbuch schon etwas aus.», p. 85.

6. - «Die Mitternacht beginnt nicht um 0 , sie beginnt um 24 Uhr, das ist es... Der alte Tag schleift in den neuen, der dauerlose Zeitpunkt stockt eine volle Stunde, heute bleibt erst mal gestern - da ächzen die Toten in ihren Gräbern, und die wachsamen, furchtsamen Hunde heulen.» (p. 8. Les références de page sans mention de titre renvoient à Zweiundzwanzig Tage... Rostock: VEB Hinstorff Verlag, 1973). Dans son essai Vor Feuer Schlünden. Erfahrung mit Georg Trakls Gedicht, Rostock: VEB Hinstorff Verlag 1984, Fühmann revient sur l'expérience fondatrice du minuit: la découverte du poème Untergang, au début du mois de mai 1945, est une illumination pour le jeune soldat nazi : la révélation de l'inéluctabilité de la défaite et la plongée dans l'angoisse existentielle, cf. le commentaire du vers «O mein Bruder klimmen wir blinde Zeiger gen Mitternacht», pp. 23-24, 29-31: «... die Mitternacht [ist ...] das Heraustreten einer besonderen Stunde aus dem gleichförmigen Stundenfluß, der Wellenberg einer Gegen-Zeit zu der als einzig geltend tradierten, da als Verkehrung aller dort gültigen Werte eine Gegen-Welt sich auftut: der schwefellichtige Gegen-Mittag, da Satan im Kreis der Hexen erscheint, um vorerst bis zum Glockenschlag eins oder auch bis zum Hahnenschrei sein Reich zu errichten, das schon in der 
Welt ist und doch nicht in ihr ist: in und zu dieser Einen das Andere. [...] Die Mitternacht war uns das schwarze Tor, zu dem wir klommen, um ins Nichts zu stürzen, in die Finsternis der Finsternis, die alles Hoffen und Glauben löscht...», loc. cit., p. 31. La confrontation entre le monde paradisiaque et celui des terreurs nocturnes est une première figure de l'opposition entre le conte et le mythe, thématisée dans la dernière partie du journal, qui annonce l'essai.

7. - La même tension affecte le commencement de Das Judenauto, comme nous avons tenté de le montrer dans notre article «Franz Fühmann Das Judenauto. La "leçon" d'une enfance allemande?", in : Germanica ${ }^{\circ}$ XV, Lille 1994, 83-101.

8. - A l'objection : «Was du siehst, ist ja nur die Oberfläche!», le narrateur du Journal répond: «Gewiß, aber was sonst könnte man denn sehen! Man muß nur bereit sein, sie überhaupt wahrzunehmen und als wesentlich, weil eben als Oberfläche des Wesens, anzuerkennen, dann sagt sie, wenn man sie mustert, vieles und weist, wenn man sie durchdenkt, fast auf alles hin.» (p. 16, c'est moi qui souligne).

9. - P. 21.

10. - P. 23.

11. - La tension entre la vie et la littérature, qui structure l'écriture, et particulièrement l'écriture autobiographique, ne doit pas, aux yeux de Fühmann, se résoudre au bénéfice exclusif de la « littérature ", et l'auteur envisage même à l'occasion de sacrifier la littérature. En témoigne la destruction du journal qu'il avait commencé sur sa petite-fille : «Ein Stück Leben haben, das sich nicht in Tinte auflöst, ohne jeden Hintergedanken literarischer Utilität» (p. 175) La remise en cause, ou du moins en perspective, de la littérature par la vie, est le critère de la « liberté » de l'écrivain, de l'authenticité de son écriture. Le journal de Budapest figure la sortie de la « littérature ». Le paradoxe est que l'épreuve de la vie, poussée assez loin, ramène à la littérature, sur d'autres chemins.

12. - Nous ne citerons que les remarques sur l'absence surprenante de majuscule à «ander» en contrepoint des notations sur les décalages culturels (p. 31), car elles se rattachent intrinsèquement à la thématique centrale de la Wandlung.

13. - Cette scène est encore l'occasion de réfléchir à la structure d'une histoire ouverte. «In dieser Geschichte ist alles denkbar, sie ist nach allen Seiten offen, jedes Ende ist möglich, von der Posse bis zum Mord.» (p. 105). Ces remarques prendront consistance lorsque l'auteur du journal s'interroge sur les points nodaux à partir desquels s'infléchit le cours d'une existence, de la sienne.

14. - «... Mein Gartenschlauch war über Nacht eingefroren, und wenn man da den Haupthahn aufdreht, dann passiert zunächst einmal gar nichts außer einem Stöhnen und Rütteln, dann fängt's an zu tröpfeln; dann kommt plötzlich ein ungeheurer Schwall heraus und schwemmt alles mögliche von Festgefrornem mit sich, und dann kommt eine Periode, wo das Wasser stetig und ruhig fließt. Die «Zweiundzwanzig Tage» sind das Buch einer Übergangsperiode, da brach plötzlieh etwas heraus, und in dem Schwall sind auch Formulierungen und Fragestellungen enthalten, die auf die unmittelbar zurückliegende Zeit bezogen sicherlich ihre Berechtigung haben, über die man aber hinauskommen möchte.», «Gespräch mit Jacqueline Benker-Grenz» (1979), in : Essays, Gespräche, Aufsätze 1964-1981, Rostock: Hinstorff Verlag, 1983, p. 410.

15. - PP. 57-63. Le 21.10, notons-le, est le septième jour.

16. - «So, vor dieser Stadt, am Wasser, auf den Steinen, ein paar angelnde Kinder, die mich nicht beachten, zur Seite: hier wäre ein guter Ort, Bilanz zu ziehen. In ein paar Wochen werde ich fünfzig. Was habe ich eigentlich vorzuweisen? Wie habe ich meine Teilfunktion versorgt» (p. 78). 17. - Das Judenauto représente la réalisation sans doute indépassable de la remémoration. Chaque séquence se structure autour d'un souvenir traité comme moment arraché à la durée (c'est la temporalité même de l'expérience du minuit), instant de révélation ou d'illumination.

18. - «Die Reise ins versperrte Land der Erinnerungen antreten, ins wahr- hafte Tibet - will man das wagen? Im Traum schaue ich ja schon über die Grenze. Will ich» p. 187. 
19. - Sur le concept de Wandlung, on consultera le livre de Uwe Wittstock, Über die Unfähigkeit zu trauern. Das Bild der Wandlung im Prosawerk von Christa Wolf und Franz Fühmann, Francfort: Athenäum Verlag, 1987.

20. - «Sie [Die Wandlung] ist die Erfahrung meines Lebens, sie ist seit zwanzig Jahren mein Thema, aber sie ist es eigentlich noch immer als Vorsatz, geleistet habe ich dazu bestenfalls Vorarbeiten! Ich habe das Vorher geschildert, ein wenig das Nachher, aber der entscheidende Prozeß, eben der der Wandlung, ist literarisch nicht bewältigt.» (p. 92, c'est moi qui souligne) On remarquera que ce jugement négatif sur Das Judenauto néglige la force de structuration du «moment ». Si les différentes stations de ce parcours autobiographique ne saisissent pas le processus de métamorphose qui leur conférerait une unité supérieure, certaines d'entre elles suggèrent avec une rare puissance la réalité opaque de la couche profonde du psychisme qui est le support de toutes les révolutions volcaniques de l'être. Wulf Köpke relève cet art de la visualisation d'images fortes, apocalyptiques, qui a pour corrélatif, selon lui, la difficulté d'inscrire ses textes dans une temporalité épique: «Das Momentane, das Vertikale, der ekstatische Augenblick sind seine Sache; er bleibt eben doch Lyriker», «"... wie ein Feuerstrom in mein Bewußtsein getreten..." Franz Fühmann und die Bewältigung der Zeit», literatur für leser 93/2, Frankfurt: Peter Lang, pp. 69-80, loc. cit., p. 75.

21. - La critique d'un de ses poèmes lors d'une lecture publique le trouble, en lui imposant l'idée d'être passé « en fasciste » superficiellement converti au nouvel ordre de la société communiste, cf. p. 189.

22. - «Du kannst tun, was du willst, du kommst von Auschwitz nicht mehr los», p. 195.

23. - «Wie könnte ich je sagen, ich hätte meine Vergangenheit bewältigt, wenn ich den Zufall, der sie gnädig beherrschte, zum obersten Schiedsrichter über mich setze. Die Vergangenheit bewältigen heißt, die Frage nach jeder Möglichkeit und also auch nach der äußersten stellen» (p. 198).

24. - L'énumération par laquelle l'écrivain tente d'attraper ce qu'il ne peut plus fixer s'achève par «nichts kannst du fassen», p. 220 ; le diable lui montre en ricanant «die schattengefüllten gehöhlten Hände; verlorene, unwiederbringlich verlorene Zeit. ... die Richtung ändern, aber habe ich noch die Kraft dazu?», p. 223.

25. - «... ein netter Mittag, ein lustiger Abschied, und in ein paar Wochen werde ich fünfzig, und weiter ist gar nichts, und es wird langsam kalt» (p. 224). On notera ici, comme d'ailleurs dans tout le passage qui précède, l'emploi systématique de «und», qui établit un lien de coordination lâche entre des notations disparates.

26. - «Aber wie vollstreckt man die Exekution seiner selbst?» (p. 199)

27. - «Aus meiner Haut werde ich nicht mehr können und konnte ich nie. Aber in ihr steckend: das Möglichste daraus machen, den Mut zu allen ihren Möglichkeiten haben, und das wäre bei meinem böhmischen Erbe der Mut zum Schießenlassen der Phantasie, der Mut zum Barocken, der Mut zum Traum und Paradoxen» (p.190). Sur cette évolution de Fühmann, qui s'inscrit dans la filiation $\mathrm{du}$ romantisme, cf. Hans-Georg Werner, «Romantische Traditionen in epischen Werken der neueren DDR-Literatur. Franz Fühmann und Christa Wolf», Zeitschrift für Germanistik, H. 4/80, pp. 398-416.

28. - La place nous manque ici pour analyser la fonction du mythe dans la pensée et l'œuvre du dernier Fühmann. Dans le cadre des réflexions esquissées ici, nous nous bornerons à relever que la structure du mythe, pour Fühmann, repose sur la temporalité de la métamorphose (et le suspens du temps chronologique). Elle est donc le médium de la figuration de la condition humaine dans la dialectique entre identité et métamorphose. 


\section{RÉSUMÉS}

Dans 22 Tage, c'est par la forme du journal que Fühmann accède à la dimension de l'autobiographie. Ce détour apparaît comme une médiation nécessaire pour dépasser les limites de Das Judenauto : ce premier recueil, qui figure l'évolution du nazisme au communisme comme un itinéraire de formation, s'il est fondé sur l'authenticité du souvenir personnel, est impuissant à figurer le processus de la métamorphose. Le journal des 22 jours marque une rupture et un tournant. Fühmann renonce à figurer et à élucider un passé définitivement enfoui, et toujours menaçant, mais non pas à poser la question de sa situation et de son identité d'écrivain. Le journal, qui traite les impressions de surface et expérimente des ressources nouvelles, fixe la situation existentielle du milieu de la vie, en assignant à l'écriture la fonction de réaliser son identité par la littérature.

In 22 Tage stößt Fühmann über die Tagebuchform zur Autobiographie vor. Dieser Umweg erscheint als notwendiger Schritt, um über Das Judenauto hinauszugehen. Diese erste autobiographische Sammlung, die die Entwicklung vom Nazismus zum Kommunismus als Bildungsweg konstruiert, ist zwar auf die Authentizität der persönlichen Erinnerung gegründet, doch der eigentliche Prozeß seiner persönlichen «Wandlung » wird nicht gestaltet. Das Tagebuch markiert einen Bruch und einen Wendepunkt in Fühmanns Schaffen. Fühmann verzichtet nunmehr darauf, einer endgültig verschütteten, doch immer bedrohlichen Vergangenheit nachzuforschen, besteht jedoch darauf, die Frage nach seiner Situation und seiner Identität als Dichter zu klären. Das Tagebuch, das von den Eindrücken der Oberfläche ausgeht und mit neuen Möglichkeiten des Schreibens experimentiert, bestimmt die existentielle Lage der «Hälfte des Lebens» und weist dem Schreiben die Funktion zu, seine Identität durch die Literatur zu realisieren.

\section{AUTEUR}

\section{ANNE LAGNY}

Université Charles-de-Gaulle - Lille III 